\title{
THREEFOLD SYMMETRIC MAGNETIC TWO-ION COUPLING IN HCP RARE-EARTH METALS
}

\author{
J. JENSEN \\ Ørsted Laboratory, Niels Bohr Institute \\ Universitetsparken 5, 2100 Copenhagen, Denmark
}

\begin{abstract}
The heavy rare earths crystallize in the hcp structure. Most of the magnetic couplings between two ions in these metals are independent of the two different orientations of the hexagonal layers. However, trigonal anisotropy terms may occur, reflecting that the $c$-axis is only a threefold axis. In the presence of a trigonal coupling the symmetry is reduced, and the double-zone representation in the $c$-direction ceases to be valid. The strong interaction between the transverse optical phonons and the acoustic spin waves propagating in the $c$-direction of $\mathrm{Tb}$ detected more than twenty years ago, was the first example of a trigonal coupling found in these systems. A few years ago a careful neutron-diffraction study of the $c$-axis modulated magnetic structures in Er showed the presence of higher harmonics at positions along the $c$-axis translated by odd multiple of $2 \pi / c$. This indicates distortions of the structures due to trigonal couplings, and the same characteristic phenomenon has now also been observed in Ho. Additionally, mean-field calculations show that a trigonal coupling in $\mathrm{Ho}$ is required, in order to explain the increase in the commensurable effects observed for the 8 and 10 layered periodic structures, when a field is applied along the $c$-axis.
\end{abstract}

PACS numbers: 75.10.-b, 75.25.+z, 75.30.Gw

\section{Introduction}

The dominant magnetic couplings in the rare-earth metals are the single-ion anisotropy terms due to the crystalline electric field acting on the magnetic $4 f$ -electrons, and the Ruderman-Kittel-Kasuya-Yoshida RKKY-exchange interaction by which the $4 f$-moments on pairs of ions are coupled indirectly through the intermediary of the conduction electrons. To a first approximation, assuming the conduction electrons to behave as free electrons, the indirect exchange leads effectively to a Heisenberg Hamiltonian for the coupling between the $4 f$-moments. The conduction electrons at the Fermi surfaces of the rare-earth metals are predominantly $d$-electron-like, and orbital modifications of the exchange coupling introduce anisotropic two-ion couplings in the magnetic Hamiltonian in addition to the Heisenberg interaction. Generally, it is not easy to separate two-ion anisotropy 
effects from those due to the single-ion crystal-field terms. The most direct identification of anisotropy effects caused by the two-ion terms is based on symmetry considerations. For example, the single-ion anisotropy and the Heisenberg exchange may occasionally leave accidental degeneracies in the magnetic excitation spectrum, which may be lifted only if anisotropic two-ion couplings are present. This happens for the spin waves at the $K-H$ edge in the Brillouin-zone of ferromagnetic $\mathrm{Tb}$, and for the singlet-doublet excitations in the basal-plane of paramagnetic Pr. Additionally, there is the indirect but weighty evidence that anisotropic components of the two-ion couplings are required in order to explain the excitation spectra in $\mathrm{Tb}$ and $\mathrm{Er}$, see the reviews [1,2]. The indications are that the coupling between the different moments in the rare-earth metals involves a great variety of anisotropic interactions besides the isotropic Heisenberg interaction. The only limitation seems to be the one set by symmetry. This is, however, illusory to some extent as the symmetry of the magnetic ground state may be lower than that given by the $4 f$-moments, involving as it does a specification of the moments due to the conduction electrons.

The heavy rare-earth metals are all hexagonal close-packed and the magnetically ordered phases of the $4 f$-moments found in these metals are either the ferromagnet or they are antiferromagnetically modulated along the $c$-axis. In these structures the individual hexagonal layers are uniformly magnetized in a direction which in the antiferromagnetic case changes from one layer to the next. The uniformity of each hexagonal layer means that the IIarniltonian has to obey the symmetry operations which leave the hexagonal layers unchanged. The general Hamiltonian for the $4 f$-moments valid in this situation has been derived from symmetry arguments $[3,4]$. The possible contributions may be divided into two classes. The first one comprises terms which are invariant with respect to either an arbitrary rotation or to a rotation by $60^{\circ}$ of the coordinate system around the $c$-axis. The second class contains terms which reflect the fact that the $c$-axis is only a threefold symmetry axis of the lattice; the trigonal couplings. The terms of principal importance, the single-ion anisotropy and the Heisenberg exchange all belong to the first class. These terms do not depend on the two orientations of the hexagonal layers if each layer is uniformly magnetized, corresponding to an effective periodicity of the lattice of $c / 2$ instead of $c$ along the $c$-axis, or an effective reciprocal lattice vector of length $4 \pi / c$. If it is found that this double-zone representation does not apply, then it may be deduced that significant trigonal couplings are present as they are the only ones which may distinguish between the two sublattices. Effects due to trigonal couplings have now been positively identified in three of the heavy rare-earth metals, and in the following we shall review these indications found in $\mathrm{Tb}, \mathrm{Ho}$, and Er.

\section{The magnon-phonon interaction in terbium}

$\mathrm{Tb}$ is ferromagnetic below $T_{\mathrm{C}} \approx 220 \mathrm{~K}$ with the moments lying in the basal plane along a $b$-direction. The properties of the spin waves in this phase have been studied experimentally in great detail by inelastic neutron scattering [3]. The dispersion relation of the excitations observed at $53 \mathrm{~K}$ with the scattering vector along the $c$-axis is shown in Fig. 1. The results are plotted in the reduced zone showing 


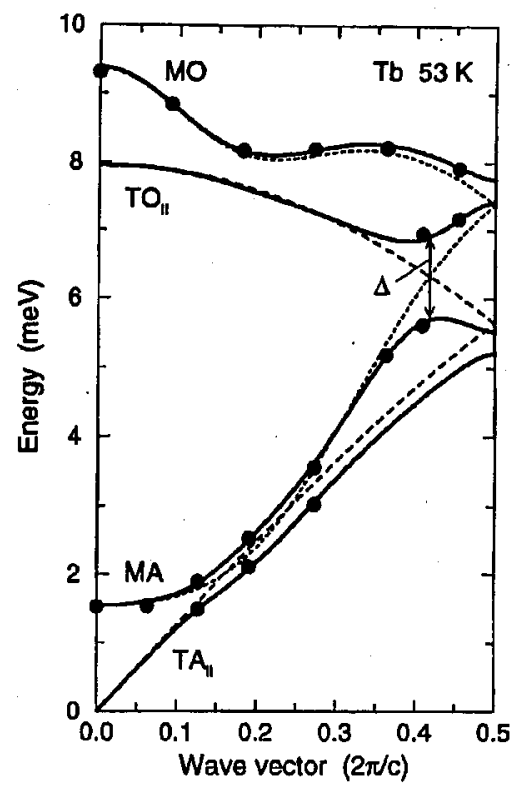

Fig. 1. The dispersion relations for the magnons and phonons propagating in the $c$-direction of $\mathrm{Tb}$ at $53 \mathrm{~K}$. The normal modes (solid lines) are mixed magnon-phonon states, and energy gaps appear at the crossing points of the unperturbed dispersion relations (dashed lines). The acoustic magnons interact both with the acoustic and the optical transverse phonons polarized parallel with the magnetization.

that the acoustic magnons (MA) interact both with the acoustic $\left(\mathrm{TA}_{\|}\right)$and the optical $\left(\mathrm{TO}_{\|}\right)$phonons. Hybridization effects due to linear couplings between the magnons and phonons have been observed at several places in the Brillouin zone, nearly every time a magnon branch happens to be close to a phonon branch.. The strongest one observed is the acoustic-optical resonance producing the energy gap $\Delta$ shown in Fig. 1, which was detected for the first time by Nielsen et al. [5]. The phonons which are coupled to the magnons in Fig. 1 are in both cases the transverse ones polarized parallel with the magnetization vector [3]. The neutron cross-section of these phonons vanishes when the scattering vector is along the $c$-axis, and the peaks due to the normal modes are only observable due to the magnon part of their state vectors, which is larger the closer the normal mode is to the unperturbed magnon branch (indicated by the short dashed line in Fig. 1). The magnon-phonon interactions have also been studied in the phase where the moments have been rotated by an external field so to be along an $a$-axis. The magnitudes of the couplings are found to be the same, but whereas the normal coupling still involves the MA and the $\mathrm{TA}_{\|}$modes, the acoustic-optical coupling now occurs between the magnons (MA) and the optical transverse phonons polarized perpendicular to the magnetization vector $\left(\mathrm{TO}_{\perp}\right)$.

Group theoretical arguments [6] show that the double-zone representation is valid for the pure phonons, so the acoustic-optical coupling may only derive 
from the magnetic part of the Hamiltonian, and as discussed in the introduction this is an unambiguous indication for the presence of a trigonal coupling. Including these couplings in the Hamiltonian it is found that they may indeed produce an acoustic-optical interaction between the excitations propagating along the $c$-axis. The selection rules determined by the symmetry elements of the $4 f$-moment system have been derived in two independent ways $[3,6]$. They show that an acoustic-optical interaction is only allowed between the MA mode and the $\mathrm{TO}_{\perp}$ mode in the $b$-axis magnet and between the MA and the $\mathrm{TO}_{\|}$modes in the $a$-axis magnet, corresponding to the observations if the two cases are interchanged. Thus the neutron scattering results for the acoustic-optical magnon-phonon interaction in the $c$-direction of $\mathrm{Tb}$, are in disagreement with the general results based on the symmetry of the $4 f$-moment system.

The acoustic-optical coupling derives from a two-ion coupling which depends on the displacements of the ions. Because all the relevant two-ion couplings in these metals are due to the conduction electrons, the violation of the selection rules must be caused by these electrons. As discussed by Liu [7] all the energy levels of the band electrons are degenerate across the Brillouin zone surface $A H L$ if the spin-orbit coupling is neglected. The spin-orbit coupling lifts the degeneracies in this plane (except along the line $A L$ ) and thus enables the electrons to distinguish between the two sublattices. The conduction electrons may mediate trigonal couplings, but only because the band-electron states are modified by the relativistic spin-orbit coupling. In the ordered phase, where the conduction electrons are polarized by the exchange field of the $4 f$-moments, the spin-orbit coupling also gives rise to a mixing of the spin states of the band electrons, so that they are not pure spin up or spin down states relative to the direction of the $4 f$-moments. The spin-density wave of the conduction electrons induced by the $4 f$-moments is not necessarily polarized collinear with these moments. Although the average polarization of the conduction electrons is parallel to the $4 f$-moments, the direction of the polarization vector may vary in space within the single unit cells. The properties of the strong magnon-phonon interaction in the $c$-direction of $\mathrm{Tb}$ can only be explained in one way. It has to involve the spin-orbit coupled band electrons to allow the coupling to occur between acoustic and optical modes, as proposed by Liu [7]. In addition it must depend in a direct way on the oscillating perpendicular component of the spin-density wave, in order to explain why the polarization of the transverse phonons coupled to the magnons is the opposite of that predicted by the general symmetry arguments above. This remarkable conclusion was reached about twenty years ago [3], and it deserves more attention than it has been given hitherto.

The equations of motion of the coupled magnon-phonon system are analyzed in Ref. [3]. The behaviour of the normal modes depends slightly on whether the perpendicular component of the conduction-electron spins is assumed parallel or perpendicular to the $c$-direction. In the first case the four coupled modes in the $b$-axis magnet combine into two doubly degenerate modes at $A$, whereas this degeneracy is removed if the spin component perpendicular to the $4 f$-moments is along the $a$-axis. In Fig. 1 we have used the latter condition as it seems to be the best choice in the comparison with the experiments. However, experimentally it 
was not possible directly to resolve the energy gap at $A$ of about $0.3 \mathrm{meV}$, because the intrinsic width of the spin waves is about $0.5 \mathrm{meV}$ at this wave vector [3].

There are some indications of similar acoustic-optical magnon-phonon interaction in the cone phase of Er, see Fig. 6.2 in Ref. [1]. The magnetic properties of Dy and $\mathrm{Tb}$ are very similar, except that $\mathrm{Dy}$ is ferromagnetically ordered along an $a$-axis instead of a $b$-axis. The hybridization of the magnon and phonon excitations observed at $4.7 \mathrm{~K}$ along the $c$-axis in Dy [8] may be explained in terms of the normal coupling (with a magnitude of about $60 \%$ of the coupling in $\mathrm{Tb}$, corresponding to a scaling proportional to the Stevens factor for a quadrupole coupling). This indicates that the possible acoustic-optical coupling in Dy is at least a factor of 3 smaller than the coupling in $\mathrm{Tb}$.

\section{The wobbling cycloid in erbium}

In Er there are three distinctly different magnetic phases, as reviewed by Koehler [9]. The phase between $T_{\mathrm{N}} \approx 84 \mathrm{~K}$ and $T_{\mathrm{N}}^{\prime} \approx 52 \mathrm{~K}$ is a sinusoidal longitudinal phase with the ordering vector $\tau \approx \frac{2}{7} \tau_{c}$, corresponding to a period of approximately 7 layers $\left(\tau_{c}\right.$ is the reciprocal lattice vector along the $c$-axis of length $\left.\tau_{\mathrm{c}}=2 \pi / c\right)$. Between $T_{\mathrm{N}}^{\prime}$ and $T_{\mathrm{C}} \approx 18 \mathrm{~K}$ there is a modulated ordering of both the longitudinal and the basal-plane components of the moments, and $\tau$ decreases from $\frac{2}{7} \tau_{\mathrm{c}}$ to $\frac{1}{4} \tau_{\mathrm{c}}$. At $T_{\mathrm{C}}$ there is a first-order transition to a cone phase which has a ferromagnetic moment along the $c$-axis and a helical ordered basal-plane component at $\tau \approx \frac{5}{21} \tau_{\mathrm{c}}$.

If anisotropy effects can be neglected, the two-ion exchange leads to a modulated structure with the wave vector $\tau$ at which the exchange coupling has its maximum. The free energy of the modulated structure is minimized when all the moments are parallel to one plane and have a constant length. Hence the ordered structure favoured by the exchange is circularly polarized, corresponding to a helix if the plane determined by the moments is perpendicular to $\tau$, or a cycloid if $\tau$ is parallel to this plane. The orientation of the plane spanned by the moments is determined by the anisotropy terms. In the rare earths the dominant one is the single-ion quadrupole coupling, and the sign of this term defines the system to be either an easy-planar system like $\mathrm{Tb}$ or Ho, or an easy $c$-axis system like Er or Tm. The ordering vector in these systems is along the $c$-axis, and in the easy-planar case the helical ordering minimizes at the same time the free-energy contributions from the exchange coupling and from the anisotropy (neglecting a relatively weak anisotropy within the basal plane). In the easy $c$-axis systems the anisotropy energy is minimized when the moments are aligned parallel or antiparallel to the $c$-axis, which is only satisfied partly by the cycloidal structure preferred by the exchange coupling. The requirement that the length of the ordered moments should stay about constant is the more relaxed the higher the temperature is, and the magnetic phase just below $T_{\mathrm{N}}$ is longitudinally polarized with the length of the moments changing sinusoidally along the $c$-axis. At decreasing temperatures higher-order $(2 n+1)$-harmonics develop (proportionally to $\left(T-T_{\mathrm{N}}^{(2 n+1) / 2}\right)$ in the mean-field approximation), corresponding to a "squaring up". of the variation of the length of the moments. If the axial anisotropy is very large, the system stays in the longitudinal phase at all temperatures below $T_{\mathrm{N}}$, as happens in $\mathrm{Tm}$, where the 
ordered moments end up as a commensurable square wave in the low temperature limit. The higher-order odd harmonics are not favourable for the exchange energy. In the case of $\mathrm{Er}$, where the ratio between the axial anisotropy and the exchange energies is smaller than in Tm, the system is driven into a compromise at $T_{\mathrm{N}}^{\prime}$ between the two competing terms in the free energy, namely the elliptically polarized cycloidal structure. In this structure the moments approach their saturation value with less cost in exchange energy than the square wave, but at the expense of the anisotropy energy.

The high-resolution synchrotron X-ray studies of the intermediate cycloidal phase of Er made by Gibbs et al. [10] indicated the presence of a number of long-period commensurable structures, which they explained to be regular arrangements of 3 or 4 layers of moments with an alternating positive or negative component along the $c$-axis. The 7 layered structure observed close to $T_{\mathrm{N}}^{\prime}$ which may be designed by the (43)-structure, thus comprises 4 hexagonal planes of moments with a positive $c$-component followed by 3 planes of moments with a negative $c$-component. As the temperature is lowered, more and more triplets are replaced by quartets, until the system just above $T_{\mathrm{C}}$ only consists of quartets, which is the (44)-structure with $\tau=\frac{1}{4} \tau_{\mathrm{c}}$. The small maignitude of the magnetic X-ray cross-section makes it difficult to detect the higher harmonics with this technique. A knowledge of the higher harmonics is required for a final determination of the structures. By the use of a large single crystal and a triple-axis spectrometer for isolating the purely elastic scattered neutrons, Cowley and Jensen [4] were able to determine the intensities of most of the harmonics in the commensurable structures of Er. The experimental results were compared with the diffraction intensities of the corresponding structures predicted by a mean-field model. This comparison confirmed that the basic feature of the commensurable structures is the one proposed by Gibbs et al. Unexpectedly, the neutron experiments also showed the presence of scattering peaks along the $c$-axis at $\pm(2 n+1) \tau+m \tau_{\mathrm{c}}$ for odd integer values of $m$. These indicate that the magnetic structures depend on the two orientations of the hexagonal layers in the hcp lattice, implying that the structures are distorted by trigonal couplings. The intensity of the largest one of the extra peaks is comparable with the intensity of the normal ( $m$ even) 7 . harmonic, showing that the distortions are of some significance. The scattering peaks at odd values of $m$ may be reproduced by the mean-field model when the following trigonal coupling term is included in the Hamiltonian for the $i$-th ion

$$
\begin{aligned}
& \Delta \mathcal{H}_{\mathrm{MF}}(i \in p \text {-th plane })=(-1)^{p} \sum_{n \geq 1}\left[K_{31}^{21}\right]_{n} \\
& \quad \times\left\{\left[O_{3}^{2}(i)-\frac{1}{2}\left\langle O_{3}^{2}(i)\right\rangle\right]\left\langle J_{y}(p+n)-J_{y}(p-n)\right\rangle\right. \\
& +\left[O_{3}^{-2}(i)-\frac{1}{2}\left\langle O_{3}^{-2}(i)\right\rangle\right]\left\langle J_{x}(p+n)-J_{x}(p-n)\right\rangle \\
& -(-1)^{n}\left[J_{y i}-\frac{1}{2}\left\langle J_{y i}\right\rangle\right]\left\langle O_{3}^{2}(p+n)-O_{3}^{2}(p-n)\right\rangle
\end{aligned}
$$




$$
\left.-(-1)^{n}\left[J_{x i}-\frac{1}{2}\left\langle J_{x i}\right\rangle\right]\left\langle O_{3}^{-2}(p+n)-O_{3}^{-2}(p-n)\right\rangle\right\} .
$$

The argument $p \pm n$ denotes an ion in the uniformly magnetized ( $p \pm n)$-th hexagonal layer, and the Stevens operators $O_{3}^{ \pm 2}=\frac{1}{2}\left(J_{z} O_{2}^{ \pm 2}+O_{2}^{ \pm 2} J_{z}\right)$, where $O_{2}^{2}=J_{x}^{2}-J_{y}^{2}$ and $O_{2}^{-2}=J_{x} J_{y}+J_{y} J_{x}$. The $x-, y$, , and $z$-axes are assumed to be along the $a_{-}, b-$, and $c$-axes of the hcp lattice, respectively. In the lowest order there are three possibilities for a trigonal coupling which is consistent with the symmetry elements of the hcp lattice. The main effects of the three fourth-rank couplings are the same, but there are some quantitative differences and the coupling given by Eq. (1) is found to be the best choice in Er [4], and also in Ho [11, 12]. The coupling changes sign from one sublattice to the next, and in the case of a cycloidal structure in the $x-z$ plane (the $a-c$ plane) described by one harmonic at $\tau$, the coupling introduces a field in the $y$-direction with two periodicities given by the wave vectors $2 \tau \pm \tau+\tau_{\mathrm{c}}$. Including the higher harmonics this corresponds to the case of Er, where the hexagonal anisotropy determines the $a$-axis to be the easy planar axis. The field in the $y$-direction due solely to the trigonal coupling causes a wobbling of the cycloid out of the $a-c$ plane, and the structures predicted by the mean-field model in the cycloidal phase are all described by the expansions

$$
\begin{aligned}
& \left\langle J_{a}(p)\right\rangle=\sum_{s=1,3, \ldots}(-1)^{(s-1) / 2} A_{a}(s) \sin [s \tau p c / 2] \\
& \left\langle J_{b}(p)\right\rangle=\sum_{s=1,3, \ldots}(-1)^{(s-1) / 2} A_{b}(s) \sin \left[s\left(\tau+\tau_{\mathrm{c}}\right) \mathrm{pc} / 2\right], \\
& \left\langle J_{c}(p)\right\rangle=\sum_{s=1,3, \ldots}(-1)^{(s-1) / 2} A_{c}(s) \cos [s \tau p c / 2] .
\end{aligned}
$$

$p$ is the numbering of the layers in the period, and the sums over $s$ include terms up to half the number of layers in one commensurable period. All the amplitudes $A_{\alpha}(s)$ in the three expressions are positive. $A_{\mathrm{c}}(s)$ decreases monotonically with increasing $s$ as does $A_{a}(s)$ in general, while the behaviour of $A_{b}(s)$ is more complicated. The relative phases of the different terms are consistent with the conditions that $\left\langle J_{\mathrm{c}}\right\rangle$ approaches the square wave, $A_{\mathrm{c}}(s)=4 J / \pi s$ in the saturated case, while the $a$ and $b$ components are smallest/largest when $\left\langle J_{c}\right\rangle$ is largest/smallest. The neutron diffraction intensities determine only the amplitudes, but assuming the phases to be those predicted by the mean-field calculations, the intensities may be used for a derivation of the magnetic structures. The 38 layered 2(44443)-structure determined in this way from the experimental scattering intensities, is compared in Fig. 2 with the result predicted by the mean-field model [4]. The two structures are very similar. The main difference is that the configuration of moments obtained experimentally is somewhat more open in the $a-c$ plane than that deduced from the mean-field model.

The magnon-phonon interaction in $\mathrm{Tb}$ discussed in the preceding section, violates the conditions determined by the symmetry of the isolated $4 f$-moment system. The trigonal coupling in $\mathrm{Er}$ is undoubtedly established in the similar way as the magnon-phonon interaction in $\mathrm{Tb}$, via the spin-orbit coupled conduction electrons. One may ask whether this also implies that the trigonal coupling breaks the 

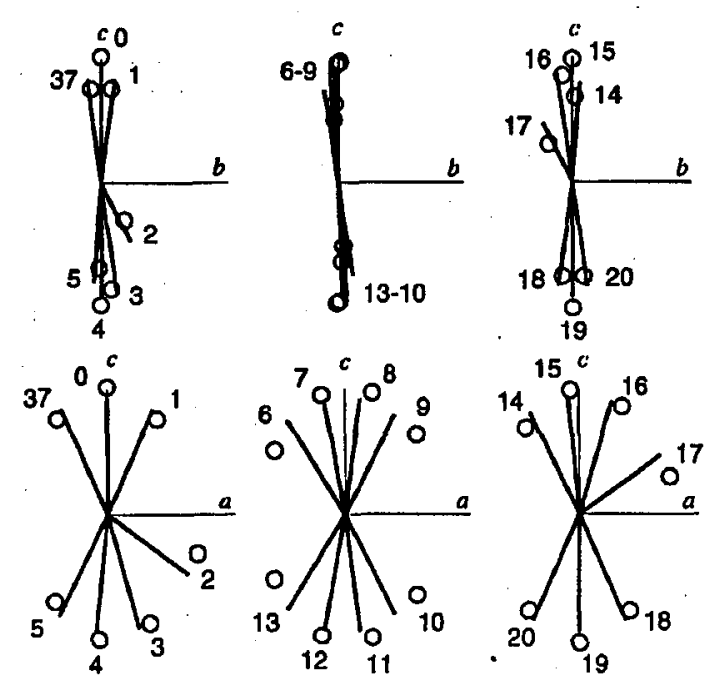

Fig. 2. The wobbling cycloidal structure in the 2(44443)-phase of Er at $29 \mathrm{~K}$. The lines are the calculated angular moments projected, respectively, onto the $b-c$ and the perpendicular $a-c$ plane in the upper and lower part of the figure. The circles are the corresponding results deduced from the experimental intensities as explained in the text. The $(a, b, c)$-axes shown are of length $J=7.5$, corresponding to the saturated $4 f$-moment, but the $b$-components have been multiplied by a factor of 2 . The moments are labelled by the numbering $p$ of the layers in the period. The second half of the 38-layered commensurable period is related to the first half of the period shown, in the way that the $b$-components are the same whereas the $a$ - and $c$-components have their signs changed.

symmetry of the local moment system in Er. The definition used above, that the $x$ and $y$-axes are respectively along the $a$ - and $b$-directions, implies that the Hamiltonian in (1) is consistent with the symmetry of the $4 f$-moment system, whereas if we interchange the definition of the two axes and assume the $x$-axis to be along a $b$-direction, the symmetry is broken in the same fashion as in Tb. In the case of a cycloidal structure in the $a-c$ plane, the symmetry-breaking trigonal coupling would produce not a dipole field but a quadrupole field in the $x$-direction perpendicular to the $y-z$ plane of the cycloid. This trigonal coupling would therefore not lead to any non-planar distortions of the cycloid at the highest temperatures below $T_{\mathrm{N}}^{\prime}$, where the field due to the trigonal coupling is weak. The mean-field calculations indicate that the modified trigonal coupling would only give rise to additional neutron-scattering peaks at temperatures well below $T_{\mathrm{N}}^{\prime}$ (below $25-30 \mathrm{~K}$ ). This is in contradiction with the experimental observations, where the non-planar distortions are seen as soon as the basal-plane moments order. In the cycloidal phase the system may choose among six orientations of the plane of the cycloid, and the presence of the different domains makes it very difficult to decide experimentally, which of the two basal-plane components corresponds to which of the neutron 
scattering peaks. However, the knowledge that the sixfold anisotropy term in $\mathrm{Er}$ determines the $a$-axis to be the easy planar axis, and that the non-planar distortion of the cycloidal structure appears immediately below $T_{\mathrm{N}}^{\prime}$, guarantees that the dominant part of the trigonal coupling in $\mathbf{E r}$ is consistent with the symmetry properties of the $4 f$-moment system. The different behaviour of the trigonal couplings in $\mathrm{Tb}$ and Er must be due to the fact that in $\mathrm{Tb}$ it is an external component (the lattice), whereas the coupling considered in $\mathrm{Er}$ is an internal one within the $4 f$-moment system.

\section{The commensurable magnetic structures in holmium}

The basal-plane moments in Ho are arranged in a helical pattern at all temperatures below $T_{\mathrm{N}} \approx 133 \mathrm{~K}$ [9]. The $c$-components order ferromagnetically at $T_{\mathrm{C}} \approx 20 \mathrm{~K}$, resulting in a conical ordering of the moments, where the opening angle approaches $80^{\circ}$ in the zero temperature limit. The wave vector of the helix decreases monotonically from about $0.28 \tau_{\mathrm{c}}$ at $T_{\mathrm{N}}$ to about $0.167 \tau_{\mathrm{c}} \approx \frac{1}{6} \boldsymbol{\tau}_{\mathrm{c}}$ at $T_{\mathrm{C}}$. Below $T_{\mathrm{C}}$ the ordering wave vector stays constant, indicating that the magnetic structure is locked to the lattice periodicity and repeats itself after every 12 hexagonal layers.

The tendency for the period of the magnetic ordered structures in the rare earths to lock into values which are commensurable with the lattice, is determined by a complex interplay between the exchange coupling and the magnetic anisotropy terms. The exchange coupling is the main factor for determining the magnetic ordering wave vector, whereas the anisotropy is decisive for the polarization of the magnetic structure. If only axial anisotropy were important in Ho the free energy would be minimized by a regularly spiraling helix in the basal plane, at the wave vector at which the exchange has its maximum. Any anisotropy within the hexagonal layers distorts the helix. The sixfold anisotropy term induces harmonics at $(6 \pm 1) \tau$ (to a first approximation) corresponding to a bunching of the moments around the easy $b$-directions, and these harmonics are clearly observed in the neutron diffraction experiments [9]. The discreteness and the finite value of the size of the local moments mean that the free energy not only depends on the amplitudes of the harmonics but also on their phases. In the incommensurable structures only the relative phases are important, whereas the anisotropy energy associated with the distortion of the simple sinusoidal ordering of the moments depends strongly on the absolute phase in the commensurable case (the "umklapp" terms). The system therefore has an extra possibility for reducing the anisotropy energy in the commensurable case in comparison with the incommensurable one. The strength of the commensurable effect then depends on how well the gain in anisotropy energy counterbalances the increase in the exchange energy, when the ordering wave vector is shifted from the value which minimizes the exchange energy to the commensurable value. In the longitudinally polarized case, Er between $T_{\mathrm{N}}$ and $T_{\mathrm{N}}^{\prime}$, it is the finite size effect of the moments rather than the anisotropy, which is responsible for the higher harmonics (the squaring up process) and thus also for the commensurable effects.

Ho was chosen by Gibbs et al. [13] as a test material for an investigation of the magnetic X-ray cross-section, using the intense radiation from a synchrotron 
source. Although the magnetic scattering intensity is weak, the narrow resolution in the X-ray experiments made it possible to detect two other commensurable structures in Ho below $25 \mathrm{~K}$, at $\tau=\frac{2}{11} \tau_{\mathrm{c}}$ and $\frac{5}{27} \tau_{\mathrm{c}}$ besides the one at $\tau=\frac{1}{6} \tau_{\mathrm{c}}$. These commensurable structures were interpreted in terms of thi: spin-slip model [13]. In the limit of very strong hexagonal anisotropy the moments would be confined to be aligned along one of the six (in the present system) $b$-directions. The 12-layered structure is then constructed from pairs of neighbouring layers with the moments along the same $b$-direction, where the moments rotate $60^{\circ}$ from one pair to the next. The periodicity may be changed by introducing regularly spaced series of spin slips, where at each spin slip a pair is replaced by a single layer. For example, the 22 layered 2/11-structure may be obtained from the 12-layered structure by introducing a spin slip after every 5 pairs, which we shall denote as the (222221)-structure. The introduction of a spin slip after every 4 pairs leads to the (22221)-structure with $\tau=\frac{5}{27} \tau_{\mathrm{c}}$. In this structure the distance between the spin slips is 9 layers creating a distortion of the lattice with this period, which was detected by Gibbs et al. [13] as charge scattering at $\frac{2}{9} \tau_{\mathrm{c}}$. The hexagonal anisotropy in Ho is large at low temperatures, and the angle between the moments in the pair layers is small but not zero. The neutron diffraction experiments [9] determined the angle between the basal-plane components in the pair layers of the 12-layered structure to be $11.6^{\circ}$ in the zero temperature limit. The high-resolution neutron scattering experiments of Cowley and Bates [14] confirmed the applicability of the spin-slip model for Ho at low temperatures, but also showed that the structures differ more and more from the ideal spin-slip structures when the system is heated, so that at $30 \mathrm{~K}$ the angle between the pair moments has increased to about $20^{\circ}$. The hexagonal anisotropy energy is proportional to approximately $\sigma^{21}$ at low temperatures, where $\sigma$ is the relative magnetization, implying that a slight decrease in $\sigma$ leads to a strong reduction of this energy. For instance $\sigma \approx 0.925$ at $40 \mathrm{~K}$, and the hexagonal anisotropy energy is reduced by a factor of 5 compared with its value in the low temperature limit. This means that the effects of the sixfold anisotropy term around $40 \mathrm{~K}$ and above this temperature, are so small that the spin-slip model may no longer be useful.

The commensurable effects at elevated temperatures in Ho have been studied by Tindall, Steinitz and collaborators, who monitored the position of the fundamental magnetic neutron diffraction peak as a function of temperature, at fields applied along the $c$-axis or along a $b$-direction, see $[15,16]$ and references therein. A field applied in the basal plane introduces a dipole anisotropy term within this plane. This low-rank term may very well explain [12] the commensurable effects observed around $75 \mathrm{~K}$ and $100 \mathrm{~K}$, when $\tau$ is close to $\frac{2}{9} \tau_{c}$ and $\frac{1}{4} \tau_{c}$ respectively, but it is difficult to understand the lock-in at $\frac{5}{18} \tau_{c}$ detected in a $b$-axis field of $30 \mathrm{kOe}$ between $126 \mathrm{~K}$ and $T_{\mathrm{N}}$. When the field is applied in the $c$-direction the Zeeman term does not introduce any anisotropy in the plane of the helix, and if only the hexagonal anisotropy were important the commensurable effects should decrease with the field (neglecting the possible effects of a small misalignment of the applied field), because the hexagonal anisotropy energy decreases faster than the exchange energy due to the helical components, when the moments are rotated out of the plane by the external field. Nevertheless, the neutron diffraction 
experiments $[15,16]$ showed that both the $\tau=\frac{1}{5} \tau_{\mathrm{c}}$ structure around $42 \mathrm{~K}$ and the $\tau=\frac{1}{4} \tau_{\mathrm{c}}$ structure around $96 \mathrm{~K}$ were stable within a couple of degrees, when a field of about $30 \mathrm{kOe}$ was applied along the $c$-axis, whereas these plateaus in the temperature variation of $\tau$ disappeared at zero field.

The trigonal coupling, Eq. (1), leads to a small out-of-plane distortion of the helix, and the first hint of the presence of this coupling in Ho was found in the neutron diffraction results of Cowley and Bates $[1,14]$. Within perturbation theory the contribution of the trigonal coupling to the free energy of the helix is of second order, whereas a first-order contribution appears if the $c$-axis moments are non-zero

$$
\Delta F \propto \sum_{p}(-1)^{p} J_{\|} J_{\perp}^{3} \cos \left(3 \phi_{p}\right)
$$

$J_{\|}$and $J_{\perp}$ are the components of the moments parallel and perpendicular to the $c$-axis, respectively, and $\phi_{p}$ is the angle which the perpendicular component of the moments in the $p$-th layer makes with the $x$ - or $a$-axis. Thus if only the trigonal anisotropy is important for the cone structure, then every second $a$-axis is an easy axis in one of the sublattices and the other three $a$-axes are the easy axes in the other sublattice. Effects due to the trigonal coupling were clearly resolved in the cone phase of Er [4], and through a careful examination [11] of the neutron diffraction spectra obtained in the presence of a $c$-axis field, it has recently been established that the trigonal coupling distorts the cone structures in Ho. The trigonal anisotropy energy is proportional to about $\sigma^{7}$ in the cone phase, implying that its importayce relatively to the hexagonal anisotropy $\left(\propto \sigma^{21}\right)$ increases rapidly with temperature. The energy also depends strongly on a $c$-axis field, so the trigonal term has the potential for being able to explain the increase in the commensurable effects observed in Ho at elevated temperatures when a field is applied along the $c$-axis.

The commensurable effects in Ho have been analyzed within the mean-field approximation [12]. The model constructed is similar to the one applied in previous numerical analyses of the magnetic structures and excitations in Ho [17], but includes also the trigonal coupling. The model gives a good account of the magnetization curves, the spin-wave energies and the neutron diffraction results at low temperatures. The intensities of the scattering peaks due to the trigonal coupling are fitted using a set of three inter-planar parameters, $\left[K_{31}^{21}\right]_{n}, n=1$, 2 and 3 , assuming the coupling to be the normal one allowed by symmetry. The RKKY-interaction depends on the polarization of the conduction electrons, leading to a temperature dependence of the interaction as indicated by the variation of the ordering wave vector. The spin wave energies have been measured at several temperatures. The results have been used for determination of the effective exchange coupling at these temperatures [17], and the exchange coupling at the intermediate temperatures is obtained by interpolation. The possible, stable or metastable, commensurable structures at a given field and temperature as determined by this model, are established by an iteration of the mean-field equations. By a comparison of the free energy of the different structures the most stable configuration may be identified. 
The model calculations predict strong commensurable effects in the spin-slip regime at low temperatures, below $35 \mathrm{~K}$, due to the hexagonal anisotropy. This regime has been carefully investigated by Cowley et al. [18] in a $c$-axis field of $10-50 \mathrm{kOe}$. In spite of a rather monotonic variation, with field or temperature, of the position of the fundamental satellite they found by measuring the position of the higher harmonics that the diffraction pattern was determined in many cases by a superposition of neutrons scattered from domains with different commensurable $\tau$-values. Small differences in $\tau$, which may be difficult to resolve at the fundamental wave vectors, are enhanced as to be distinguishable when considering for example the fifth or the seventh harmonic. In Fig. 3 there are shown the

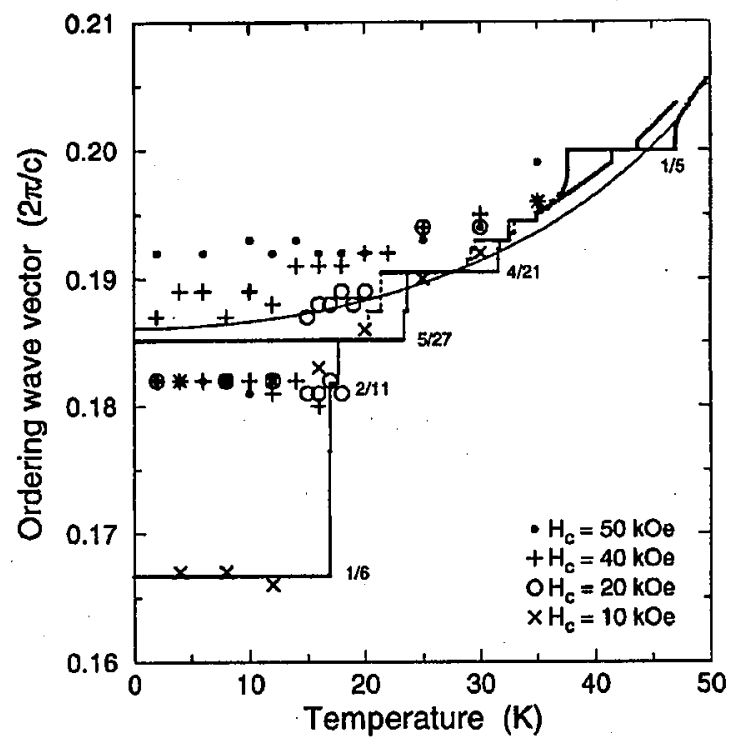

Fig. 3. The ordering wave vector in Ho as a function of temperature below $50 \mathrm{~K}$. The calculated results are shown by the horizontal solid lines connected with vertical thin solid or thin dashed lines corresponding respectively to the results obtained at zero or at a field of $50 \mathrm{kOe}$ applied along the $c$-axis. The symbols show the experimental results of Cowley et al. [18] obtained at the various values of the $c$-axis field defined in the figure. The smooth curve shown by the thin solid line is the temperature dependent position of the maximum in the exchange coupling assumed in the model.

experimental results of Cowley et al. compared with the results derived from the model calculations at zero field and in a $c$-axis field of $50 \mathrm{kOe}$. There is an overall field-dependent shift of the stable intervals of the commensurable structures towards lower temperatures, which is more pronounced the lower the temperature is. This shift is reasonably well described by the model and occurs because the moments in the spin-slip layers have a larger $c$-axis susceptibility than the moments in the pair layers, i.e. the more spin-slip layers a structure contains the more Zeeman energy it gains in a $c$-axis field. In this comparison it may be important 
that the 2/11-structure and the 3/16-structure (lying in between the 5/27- and 4/21-structures) in contrast to the neighbouring structures have a non-zero dipole moment and also a non-zero quadrupole moment in the basal plane. This means that a small misalignment of the $c$-axis field and magnetoelastic distortions would favour principally these two structures. The most important effect predicted by the mean-field model is the behaviour of the lock-in of the 10 layered $1 / 5$-structure near to $42 \mathrm{~K}$. The lock-in interval is calculated to be about $2.2 \mathrm{~K}$ at zero field and to increase rapidly to become $10 \mathrm{~K}$ at a $c$-axis field of $10 \mathrm{kOe}$, whereupon it stays more or less constant between 10 and $50 \mathrm{kOe}$. This temperature range was not included in the experiment of Cowley et al., but the effect agrees qualitatively with the observations of Tindall et al. [16], who only studied the behaviour of the first harmonic. The hysteresis effects detected by Cowley et al. may possibly explain why the lock-in intervals determined by Tindall et al. are somewhat smaller (zero at zero field and $2-3 \mathrm{~K}$ at $30 \mathrm{kOe}$ ) than predicted by the theory.

Around $100 \mathrm{~K}$ the spin-slip model no longer applies. The hexagonal anisotropy only manages to rotate the moments by about one tenth of a degree. At this temperature the ordering wave vector is close to $\frac{1}{4} \tau_{\mathrm{c}}$, but the model indicates only a marginal lock-in to the 8 layered structure. In the presence of a $c$-axis field of $30 \mathrm{kOe}$, the trigonal coupling increases the bunching effect by a factor of 4 , but the lock-in interval is still estimated to be very small, about $0.1 \mathrm{~K}$. In analogy with the fifth and seventh harmonics induced by the hexagonal anisotropy, the first-order term in the free energy due to the trigonal coupling induces a second and a fourth harmonic. Because of the factor $(-1)^{p}$ in Eq. (3) these harmonics are translated by a reciprocal lattice vector along the $c$-axis (half of a reciprocal lattice vector in the double-zone scheme), which means that the fourth harmonic appears at zero wave vector when $\tau=\frac{1}{4} \tau_{\mathrm{c}}$. In other words, in the case of a cone structure with a period of 8 layers the trigonal coupling leads to a ferromagnetic component perpendicular to the $c$-axis. Although it is small, this component has a determining effect in forming the commensurable structure. The lock-in interval increases proportionally to $\sqrt{\theta}$, where $\theta$ is the angle which the field makes with the $c$-axis, and even the slightest deviation of the field from perfect alignment along the $c$-axis will produce a sizable lock-in effect. The lock-in interval is calculated to be $2.7 \mathrm{~K}$ at $\theta=1^{\circ}$ at the field of $30 \mathrm{kOe}$. Both this value and the very weak lock-in effect at zero field are consistent with the observations made by Noakes, Tindall and collaborators [15] (the hysteresis effects discussed above should be of less importance at this high temperature). At a larger tilt angle of the field the lock-in interval is estimated to increase up to a value of $8-12 \mathrm{~K}$.

\section{Summary}

The trigonal couplings have a number of surprising effects on the magnetic properties of the rare-earth hcp metals. The acoustic-optical magnon-phonon interaction in $\mathrm{Tb}$ (and possibly also in $\mathrm{Er}$ ) is strong and depends in a direct fashion on the spin-orbit coupled band-electron states, as shown by its symmetry-breaking properties. The trigonal coupling between the $4 f$-moments derived in the cases of Er and Ho is relatively weak compared with the Heisenberg exchange coupling, but because of its low-symmetry properties, the effects due to this coupling are so distinct that they may be isolated in an unambiguous way. 
In Er the trigonal coupling produces a non-planar distortion of the cycloidal structures and probably also causes the lock-in effect at $\tau=\frac{5}{21} \tau_{\mathrm{c}}$ in the cone phase. The cone angle in $\mathrm{Er}$ is so small $\left(\approx 28^{\circ}\right)$ that the hexagonal anisotropy is unimportant. In Ho the influence of the trigonal coupling on the structures is clearly resolved in the neutron scattering experiments, and the explanation of the increased stability of the 10-layered periodic structure around $42 \mathrm{~K}$ and of the 8-layered structure around $96 \mathrm{~K}$, observed when applying a field along the $c$-axis, relies totally on the presence of the trigonal anisotropy.

The ferromagnetic structures (Gd and the low temperature phases in $\mathrm{Tb}$ and $\mathrm{Dy}$ ) or the longitudinally polarized $c$-axis modulated structures ( $\mathrm{Tm}$ and Er between $T_{N}$ and $T_{N}^{\prime}$ ) are not affected by the trigonal coupling. Therefore the only remaining candidates among the heavy rare earths to be investigated for the possible structural effects of the trigonal coupling, are $\mathrm{Tb}$ and $\mathrm{Dy}$ in their high-temperature helical phases. Of these two only Dy may be a realistic possibility, because the helical phase in $\mathrm{Tb}$ only occurs in a narrow temperature range.

The trigonal coupling in the rare-earth metals is mediated by the conduction electrons, and may thus be considered to be one of the anisotropic components of the RKKY-interaction. It occurs only because the conduction electrons are affected by the spin-orbit coupling, and because of this dependence it is the equivalent of the Dzyaloshinsky-Moriya interaction in the magnetic transition metals. The spin-orbit effects on the band electrons in the rare-earth metals are stronger than in the transition metals, and the localized moments in the $4 f$-metals (except $\mathrm{Gd}$ ) have a large orbital component. In combination the two factors may explain why the trigonal coupling in the rare-earth metals is relatively much larger than the very weak Dzyaloshinsky-Moriya interaction in the transition metals.

\section{References}

[1] J. Jensen, A.R. Mackintosh, Rare Earth Magnetism: Structures and Excitations, Oxford University Press, Oxford 1991.

[2] J. Jensen, Physica B 86-88, 32 (1977).

[3] J. Jensen, J.G. Houmann, H. Bjerrum Møller, Phys. Rev. B 12, 303 (1975); J. Jensen, J.G. Houmann, Phys. Rev. B 12, 320 (1975); J.G. Houmann, J. Jensen, P. Touborg, Phys. Rev. B 12, 332 (1975).

[4] R.A. Cowley, J. Jensen, J. Phys. Condens. Matter 4, 9673 (1992); J. Jensen, R.A. Cowley, Europhys. Lett. 21, 705 (1993).

[5] M. Nielsen, H. Bjerrum Møller, A.R. Mackintosh, J. Appl. Phys. 41, 1174 (1970).

[6] A.P. Cracknell, J. Phys. F 4, 466 (1974).

[7] S.H. Liu, Phys. Rev. Lett. 29, 793 (1972).

[8] R.M. Nicklow, N. Wakabayashi, Neutron Inelastic Scattering, IAEA, Vienna 1972, p. 603.

[9] W.C. Koehler, in: Magnetic Properties of Rare Earth Metals, Ed. R.J. Elliott, Plenum Press, London 1972, p. 81.

[10] D. Gibbs, J. Bohr, J.D. Axe, D.E. Moncton, K.L. D’Amico, Phys. Rev. B 34, 8182 (1986).

[11] J.A. Simpson, D.F. McMorrow, R.A. Cowley, D.A. Jehan, Phys. Rev. B 51, 16073 (1995). 
[12] J. Jensen, Phys. Rev. B, to be published.

[13] D. Gibbs, D.E. Moncton, K.L. D'Amico, J. Bohr, B.H. Grier, Phys. Rev. Lett. 55, 234 (1985); J. Bohr, D. Gibbs, D.E. Moncton, K.L. D'Amico, Physica A 140, 349 (1986).

[14] R.A. Cowley, S. Bates, J. Phys. C 21, 4113 (1988).

[15] D.R. Noakes, D.A. Tindall, M.O. Steinitz, N. Ali, J. Appl. Phys. 67, 5274 (1990); D.A. Tindall, M.O. Steinitz, M. Kahrizi, D.R. Noakes, N. Ali, J. Appl. Phys. 69, 5691 (1991).

[16] D.A. Tindall, M.O. Steinitz, T.M. Holden, Phys. Rev. B 47, 5463 (1993).

[17] C.C. Larsen, J. Jensen, A.R. Mackintosh, Phys. Rev. Lett. 59, 712 (1987); J. Jensen, A.R. Mackintosh, Phys. Rev. Lett. 64, 2699 (1990).

[18] R.A. Cowley, D.A. Jehan, D.F. McMorrow, G.J. McIntyre, Phys. Rev. Lett. 66, 1521 (1991). 